\title{
Comparison on seismometer sensitivity following ISO 16063-11 standard
}

\author{
Larsonnier Franck ${ }^{1}$, Rouillé Guillaume ${ }^{1}$, Bartoli Claire ${ }^{2}$, Klaus Leonard ${ }^{3}$, Begoff Philipp $^{4}$ \\ ${ }^{1}$ CEA DAM Ile-de-France, 91297 Arpajon, France \\ ${ }^{2}$ LNE, 1 rue Gaston Boissier, 75015 Paris, France \\ ${ }^{3}$ Physikalisch-Technische Bundesanstalt (PTB), Bundesallee 100, 38116 Braunschweig, Germany \\ ${ }^{4}$ SPEKTRA Schwingungstechnik und Akustik GmbH, Heidelberger Str.12, 01189 Dresden, Germany
}

Corresponding author: Franck Larsonnier, e-mail: franck.larsonnier@cea.fr

\begin{abstract}
Accelerometer calibration by primary and secondary means is now very well covered both in low and medium frequency range. This is obviously not the case for seismometers which are widely used in the world. Up to now, seismometers only have internal checks thanks to an integrated coil. Calibration of these devices address different challenges like large mass and volumes, non-rotatable devices, specific mounting. This inter laboratory comparison will help to investigate these fields and to give feedback to develop a methodology to calibrate seismometers.
\end{abstract}

Keywords: seismometer calibration, low frequency vibration, primary calibration.

\section{Introduction}

\subsection{The context}

Up to now, seismometers are only "tested" by electrical methods using an integrated calibration coil associated with the nominal transducer and a reference electrical input signal. Results derived from these measurements are useful for remote access and in situ performance, which is required as it is not planned to return the seismometers for periodic calibration, but they are not sufficient to validate the seismometer. A better confidence in seismometer calibration can be only achieved in laboratory but not on field, by a mechanical calibration by e.g. primary means as described in ISO 16063-11 [1]. Specific issues arise for mechanical calibration of these devices such as large masses and volumes, thermal, positioning effects, very high sensitivity, and a broadband frequency response from the milli Hertz up to a few hundred Hertz [2] as shown on the figure 1 .

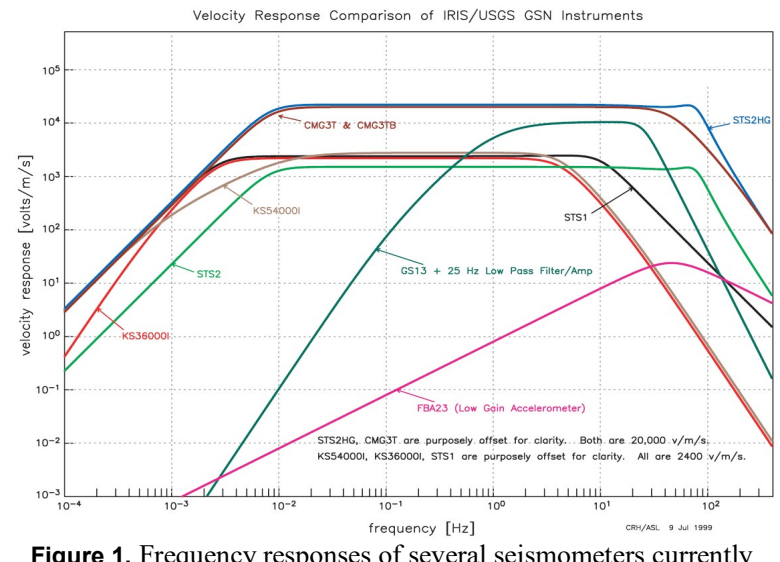

Figure 1. Frequency responses of several seismometers currently available on the market [2].
Additionally, seismometers are typically not rotatable, which means that the excitation needs to be horizontal and vertical if one wants to cover all types of seismometers. The small excitation levels lead to specific mounting configurations without fixing the sensor to the exciter table. These special features do not match with classical features of accelerometers and the corresponding calibration benches. The lack of methods for seismometer calibration and a recently developed vertical and horizontal dedicated shakers required to organise inter laboratory comparison to deal with all these side effects. The CCAUV identified the needs "Low-frequency vibration key comparison down to $0.1 \mathrm{~Hz}$ for traceability is one of the future actions to support this field" in its strategy document 2017 to 2027 [3].

The inter laboratory comparison reported here was piloted by LNE according to ISO 16063-11 with the participation of CEA, PTB and SPEKTRA all motivated in investigating in that field. The comparison included a set of two operational sensors, the CP ZM500 seismometer and the GeoSIG AC-73 accelerometer provided by CEA.

\subsection{The CP ZM500 seismometer description}

The CP ZM500 seismometer is a highly sensitive analogue vertical velocity sensor (magnet-coil transducer) designed by CEA with a mass of $11 \mathrm{~kg}$, cylindric shape of $300 \mathrm{~mm}$ height and diameter, as shown in the figure 2. Its frequency range of interest is $0.5 \mathrm{~Hz}$ to $100 \mathrm{~Hz}$. For the comparison, the sensor was selected with a nominal velocity sensitivity value of $\left.9000 \mathrm{~V} /\left(\mathrm{m}^{-1}\right)^{-1}\right)$. The theoretical response is shown on figure 3 .

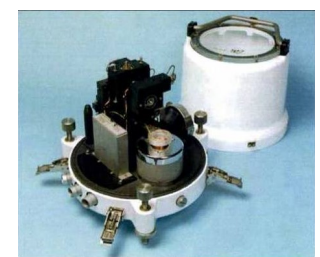

Figure 2. CP ZM500 vertical seismometer. 


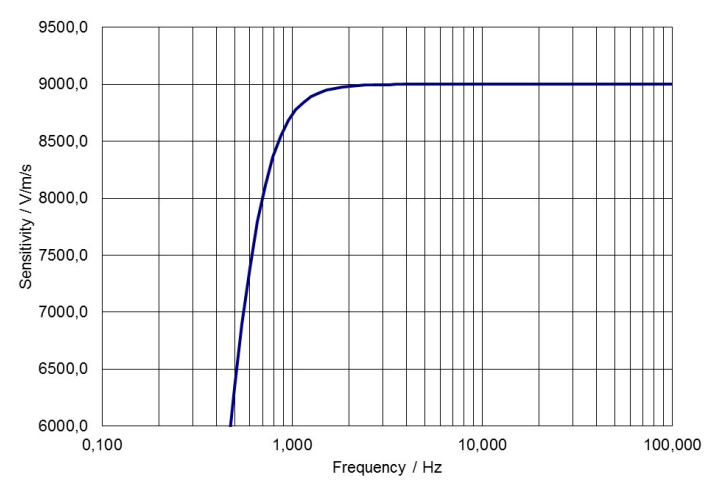

Figure 3. CP ZM500 theoretical amplitude velocity response (unit $\mathrm{V} / \mathrm{m}^{-1}{ }^{-1}$ ) as function of frequency.

\subsection{The AC-73 accelerometer description}

The AC-73 is an analogue electro-mechanical (force balance principle) triaxial downhole accelerometer made by GeoSIG as shown on figure 4. It is a broadband sensor, with a bandwidth from DC to $200 \mathrm{~Hz}$ and its weight is $3 \mathrm{~kg}$. The manufacturer specifies a sensitivity value of $5 \mathrm{~V} / \mathrm{g}$ or $510 \mathrm{mV} /\left(\mathrm{m}^{-2}\right)$ for each axis.

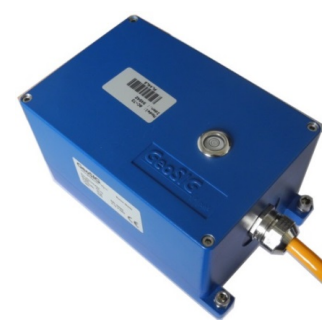

Figure 4. GeoSIG AC-73 triaxial accelerometer.

\subsection{The metrological challenges}

We can summarize the calibration as an operation that establishes a relation between a reference quantity value provided by a measurement standard and the corresponding indication of a measuring result provided by the calibrated device itself with assigned measurement uncertainties. As seismometers measure ground motions, the calibration standard should excite a known ground motion and compare it to the seismometer's electrical output signal. The application of that definition is difficult. Such a ground motion means to have a mechanical exciter to generate high quality displacement with minimal parasitic motions (yaw, roll, pitch), vibration control, and displacement or velocity measurement reference sensors accurate and traceable to SI. This enables a reliable metrological link for the calibration of seismometers.

Combining all these requirements, we need an absolute calibration method. Such a method is available and well established for small and compact accelerometers but difficult to transfer to seismometers. Consequently, to bypass this situation, up to now the external forces and accelerations for the seismometer calibration were usually transferred to internal electromechanical force generators using an in-situ calibration coil. Knowing the relation between the current in the coil and the equivalent ground acceleration, this 'calibration' becomes an electrical method. However, due to the unknow relation to the surrounding environment and missing traceability, this method is more kind of a test to check a correct function of the mechanics concerning the measuring coil of the sensor.
The comparability of the electrical calibration and methods using an exciter was investigated with CP ZM500 seismometers [4]. At low frequency the measured velocity response agrees well with the nominal value. Above $10 \mathrm{~Hz}$, the amplitude decreases strongly up to $80 \mathrm{~Hz}$ and then increases. The physical origin of this deviation is a self-capacitance and coupling capacitance phenomenon of the solenoid coils induced by the design of the function. A bias in the electrical calibration has been observed on different kind of seismometers at higher frequency regions of the response functions, which means that this method is not well suited to determine the real response of this kind of sensors.

\section{MECHANICAL CALIBRATION}

The mechanical calibration principle uses an exciter or shaker, in some configurations associated with a slip table carrying the seismometer. A reference measures the displacement or the velocity of the seismometer under test. An adjustable support may help to eliminate alignment and the Abbe errors. Parasitic vibration, thermal drift, mechanical strain should be minimised and need to be considered when estimating the measurement uncertainty.

\subsection{The SPEKTRA and CEA mechanical calibration system}

The calibrations were carried out at CEA and SPEKTRA with SPEKTRA's CS18P seismic calibration system composed of one SE-13 [5] vertical exciter, one APS 129 horizontal exciter and a laser vibrometer traceable to the SI as shown on Figure 5. The CS18P has been especially designed by SPEKTRA for primary calibration of heavy vibration sensors. The calibration frequency range is $0.1 \mathrm{~Hz}$ to $400 \mathrm{~Hz}$ for the vertical shaker and $0.1 \mathrm{~Hz}$ to $160 \mathrm{~Hz}$ for horizontal one.

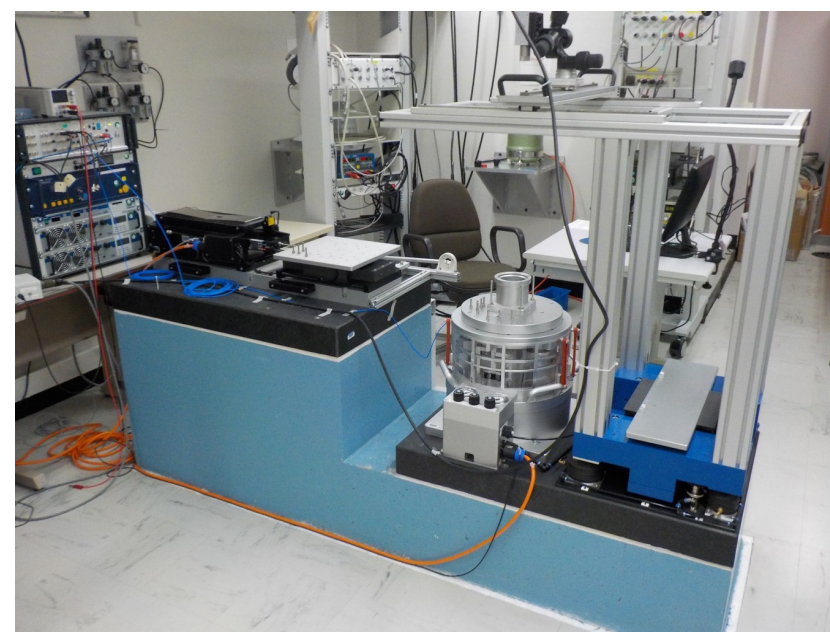

Figure 5. SPEKTRA CS18P seismic calibration system in the low frequency vibration metrology laboratory at CEA.

\subsection{The multi-component acceleration exciter of PTB}

The calibration at PTB was carried out using the multicomponent exciter [6] shown on the figure 6 . This device offers a high payload of $100 \mathrm{~kg}$ and a frequency bandwidth capability from $1000 \mathrm{~Hz}$ down to about $0.5 \mathrm{~Hz}$. The three orthogonal axes of motion can be excited simultaneously by a multi input multi output control system, which will compensate crosstalk at uniaxial excitation, too. The references for the calibration are three laser vibrometers traceable to the SI. Differently to the measuring set-ups of CEA and SPEKTRA, these will not offer a 
primary calibration, as the velocity proportional voltage outputs of the calibrated vibrometers were used.

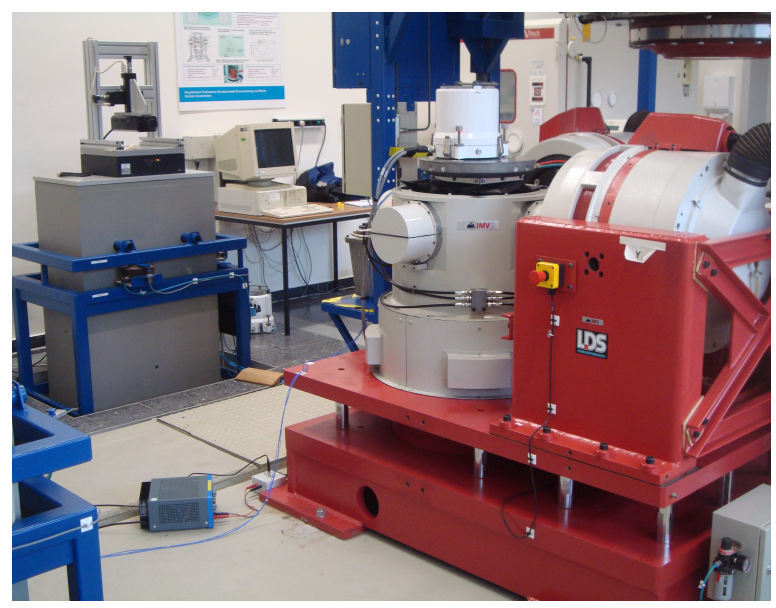

Figure 6. The multi-component acceleration exciter of PTB with the CP ZM500 on the table.

\subsection{The calibration Protocol}

A calibration protocol and a circulation organisation were defined for the set of sensors.

\subsubsection{CP ZM500 calibration protocol}

The selected measurement frequency range was $0.5 \mathrm{~Hz}$ to $100 \mathrm{~Hz}$. The measurement conditions, installations and adjustment were specially defined because PTB and SPEKTRA were not familiar with this type of sensor. The sensor was not clamped on the shaker - it was calibrated in the same way as it is implemented on field. It was adjusted in height to establish a distance of $20 \mathrm{~mm}$ between the top of the table and the base of the sensor. Horizontal setting is done using the tool associated with the sensor. We assume that the adjustment of the sensor was very similar in each laboratory during the comparison.

The calibration was carried out for three configurations. Each configuration corresponds to a specific position of the laser vibrometer's measuring spot relative to the sensor. Whatever the configuration is, the sensor which can be considered as a cylinder was kept centred with the table as shown on the figure 7 . In that position the centre of gravity of the sensor is very close to the axis of the exciter.

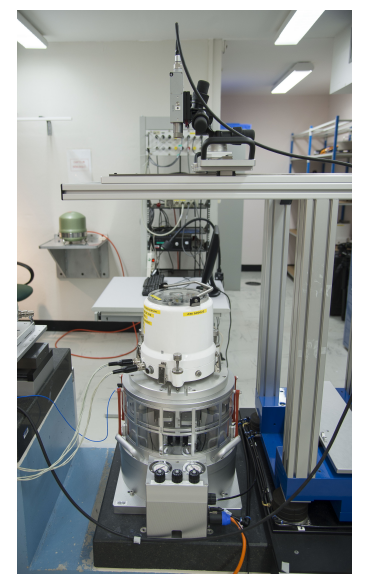

Figure 7. The CP ZM500 sensor placed on the SE-13 vibration table at CEA
The three configurations are the following as shown on figure 8: conf. A: The laser is aligned with the geometrical axis of the sensor which is close to the axis of the exciter. In that configuration the vibrometer measures the displacement directly on the top of the sensor's housing.

conf. B: The laser is aligned with the transducer axis, 5 $\mathrm{cm}$ far from the configuration $\mathrm{A}$. In that configuration the vibrometer measures the displacement on the top of the sensor.

conf. C: The laser is pointing the top of the exciter, as close as possible to the sensor at $120^{\circ}$ each other's. In that configuration the vibrometer measures directly the displacement of the shaker. This configuration is the closest to the on-site configuration.
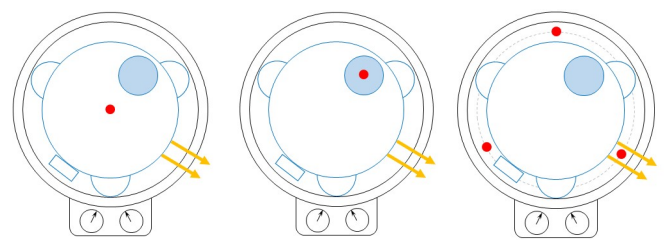

Figure 8. Top views of the configurations A, B and C (left to right) with the CP ZM500 sensor on the SE-13. The red dots indicate the laser spot locations.

\subsubsection{GeoSIG AC-73 calibration protocol}

The measurement frequency range is $0.5 \mathrm{~Hz}$ to $160 \mathrm{~Hz}$. The sensor is clamped on the shakers with a single bolt located at the rear side. For the three axes, the laser positions have been defined using a reflecting scotch tape on each side of the sensor (cf. figure 9). The deviation between the measurement positions of each laboratory is expected to be in a range of few millimeters.

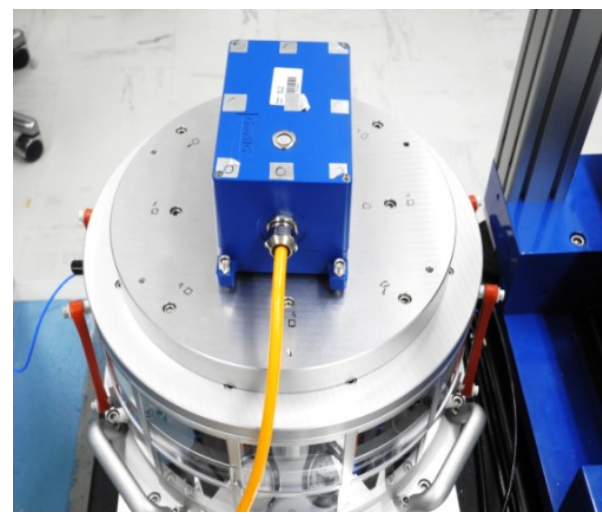

Figure 9. The AC-73 mounted on the SE-13 table.

\section{CALIBRATION RESULTS}

The calibrations of the sensors were carried out according to the protocol. The results of the two sensors will be discussed in the following sections.

\subsection{CP ZM500 calibration results}

The following figures 10, 11 and 12 show the measurements of the CP ZM500 sensitivity according to the three configurations $\mathrm{A}, \mathrm{B}$ and $\mathrm{C}$ over the range $0.5 \mathrm{~Hz}$ to $100 \mathrm{~Hz}$.

The estimated relative expanded measurement uncertainties for the derived magnitude responses at the different excitation frequencies are given in table 1. 


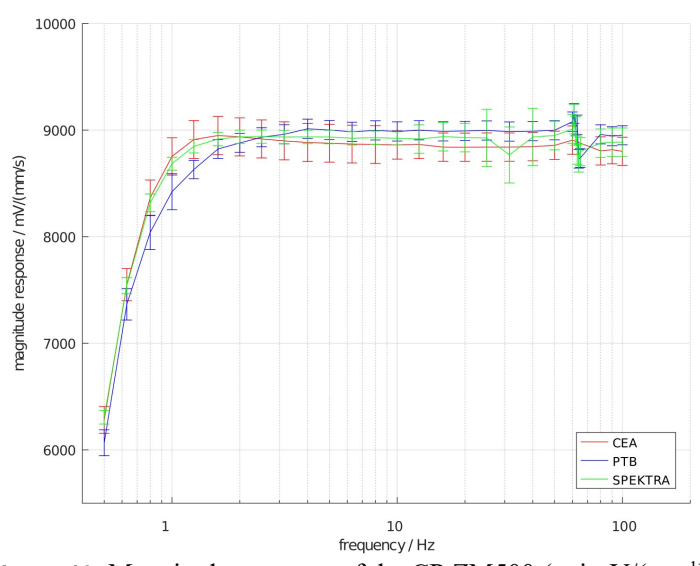

Figure 10. Magnitude response of the CP ZM500 (unit: V/(m·s ${ }^{-1}$ ) as function of the frequency in measuring configuration $\mathrm{A}$.

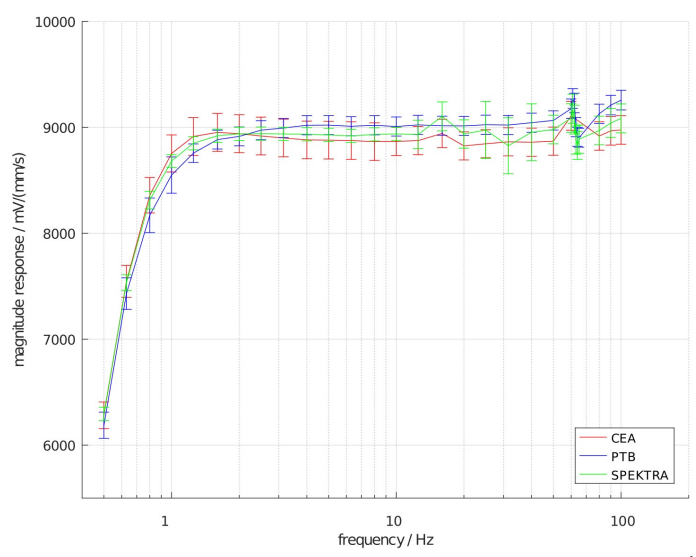

Figure 11. Magnitude response of the CP ZM500 (unit: V/(m·s $\left.{ }^{-1}\right)$ ) as function of the frequency in measuring configuration $\mathrm{B}$.

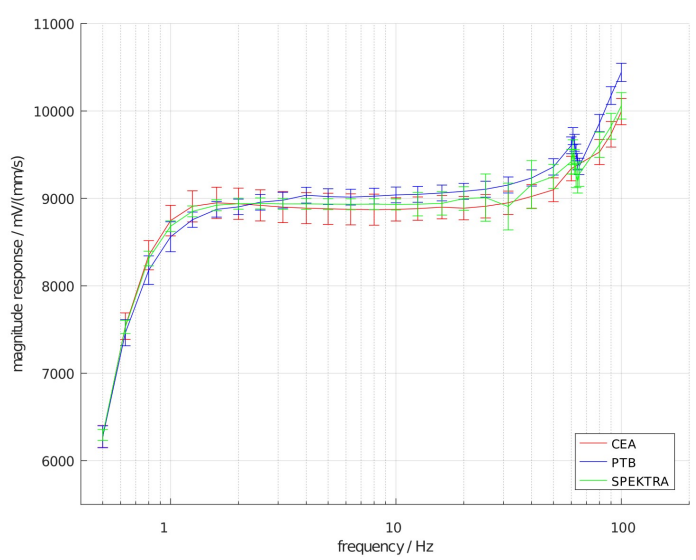

Figure 12. Magnitude response of the CP ZM500 (unit: V/(m-s $\left.{ }^{-1}\right)$ ) as function of the frequency in measuring configuration $\mathrm{C}$.

Table 1. Relative expanded measurement uncertainties $U_{\text {rel }}(k=2)$ for the magnitude response of the CP ZM500 seismometer

\begin{tabular}{l|l|l}
\multirow{2}{*}{ Participant } & \multicolumn{1}{|c}{$\begin{array}{c}\text { calibration. } \\
\text { Frequency range }\end{array}$} & \multicolumn{1}{c}{$\begin{array}{c}U_{\text {rel }}(k=2) \text { of the } \\
\text { magnitude response }\end{array}$} \\
\hline \multirow{2}{*}{ PTB } & $\leq 1 \mathrm{~Hz}$ & $2 \%$ \\
& $1.25 \mathrm{~Hz}-100 \mathrm{~Hz}$ & $1 \%$ \\
\hline \multirow{2}{*}{ CEA } & $<10 \mathrm{~Hz}$ & $2 \%$ \\
& $10 \mathrm{~Hz}-100 \mathrm{~Hz}$ & $1.5 \%$ \\
\hline \multirow{3}{*}{ SPEKTRA } & $<1 \mathrm{~Hz}$ & $1 \%$ \\
& $1 \mathrm{~Hz}-10 \mathrm{~Hz}$ & $0.7 \%$ \\
& $>10 \mathrm{~Hz}-20 \mathrm{~Hz}$ & $1.5 \%$ \\
& $>20 \mathrm{~Hz}-40 \mathrm{~Hz}$ & $3 \%$ \\
& $>40 \mathrm{~Hz}-100 \mathrm{~Hz}$ & $1.5 \%$
\end{tabular}

For further analysis we split the measured responses of the sensor in two parts depending on the frequency range for the analysis. The lower frequencies from $0.5 \mathrm{~Hz}$ to $10 \mathrm{~Hz}$ are shown in figure 13 .

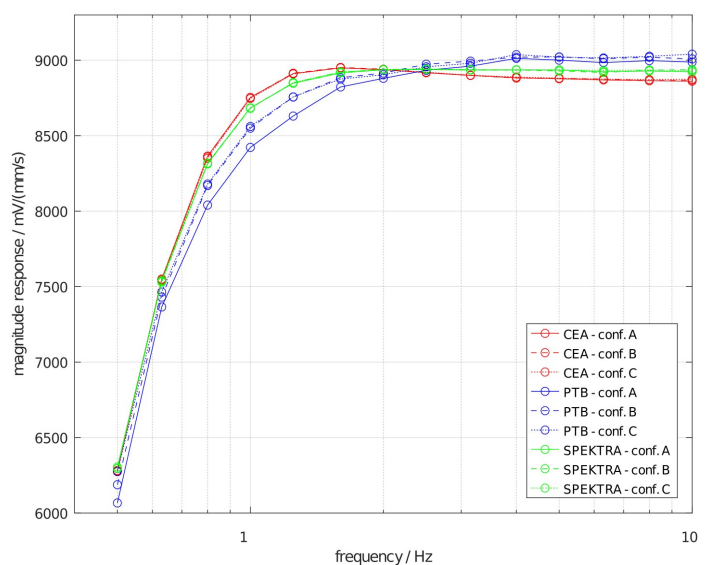

Figure 13. Magnitude response of the CPZM500 (unit: $\mathrm{V} /\left(\mathrm{m}^{-1} \mathrm{~s}^{-1}\right)$ ) over a frequency range from $0.5 \mathrm{~Hz}$ to $10 \mathrm{~Hz}$ in all three configurations.

From $0.5 \mathrm{~Hz}$ up to $1.4 \mathrm{~Hz}$, the measurements deviation of the three configurations are within $4 \%$ each other and with the theoretical response. From $1.6 \mathrm{~Hz}$ to $10 \mathrm{~Hz}$, the measurement is within $1.5 \%$ each other and with the theoretical response.

At higher frequencies from $10 \mathrm{~Hz}$ to $100 \mathrm{~Hz}$ (cf. figure 14) we can identify the following characteristics:

- At $62 \mathrm{~Hz}$, all configurations highlight the same disturbance which is not predicted with the theoretical response. Investigation identified an internal mechanical resonance of the sensor.

- For the configurations A and B, all measurements are within $\pm 2 \%$ and with the theoretical response (indicated by the orange corridor in figure 14). These configurations validate the theoretical response of the sensor without considering the actual ground movement and influences due to the stiffness of the connection between the sensor and the shaker armature.

- For the configuration $\mathrm{C}$, all measurements highlight an increasing deviation from $30 \mathrm{~Hz}$ up to $100 \mathrm{~Hz}$, up to $15 \%$ from the model at $100 \mathrm{~Hz}$. This specific deviation was historically measured by CEA, but this comparison confirms the limitation of the theoretical response depending on the sensor interaction with the ground motion at higher frequencies.

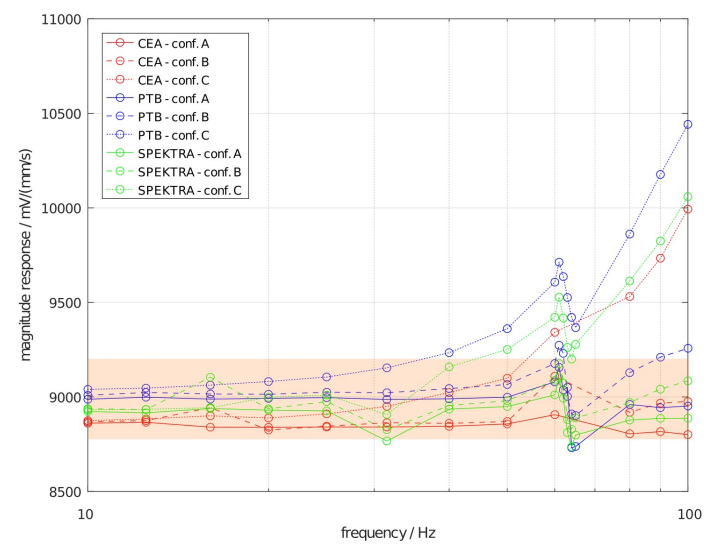

Figure 14. Comparison of the CP ZM500's magnitude response (unit: $\mathrm{V} /\left(\mathrm{m}^{-1}\right)$ ) in all three configurations over a frequency range from $10 \mathrm{~Hz}$ to $100 \mathrm{~Hz}$. Indicated in orange is the corridor within $\pm 2 \%$ of the nominal sensitivity. 


\subsection{AC-73 Calibration results}

Figures 15, 16 and 17 show the measured magnitude responses of the GeoSIG AC-73 sensitivity for the x-, yand $\mathrm{z}$-axis with assigned expanded measurement uncertainties of the three participants. The frequency range of the calibration ranged from $0.5 \mathrm{~Hz}$ to $160 \mathrm{~Hz}$ for CEA and SPEKTRA. PTB started the calibration at $1 \mathrm{~Hz}$. For the three calibrations a systematic deviations of $2 \%$ at $1 \mathrm{~Hz}$ and $1 \%$ from $1 \mathrm{~Hz}$ to $80 \mathrm{~Hz}$ appear between PTB and the two other laboratories. The maximum measurement deviations are always at $160 \mathrm{~Hz}$ even with the two CS18P.

The estimated relative expanded measurement uncertainties for the derived magnitude responses of the GeoSIG AC-73 at the different excitation frequencies are given in table 2.

Table 2. Relative expanded measurement uncertainties $U_{\text {rel }}(k=2)$ for the magnitude response of the GeoSIG AC-73 accelerometer

\begin{tabular}{l|l|l}
\multicolumn{1}{c|}{ Participant } & \multicolumn{1}{|c}{ Frequency range } & \multicolumn{1}{c}{$\begin{array}{c}U_{\text {rel }}(k=2) \text { of the } \\
\text { magnitude response }\end{array}$} \\
\hline \multirow{3}{*}{ PTB } & $\leq 1 \mathrm{~Hz}$ & $2 \%$ \\
& $1.25 \mathrm{~Hz}-100 \mathrm{~Hz}$ & $\begin{array}{l}1 \% \\
\text { higher uncertainties due } \\
\text { to type-A comp. }\end{array}$ \\
\hline \multirow{2}{*}{ CEA } & $>100 \mathrm{~Hz}$ & $1 \%$ \\
& & $0.8 \%$ \\
\hline \multirow{3}{*}{ SPEKTRA } & $10 \mathrm{~Hz}-160 \mathrm{~Hz}$ & $1 \%$ \\
& $<1 \mathrm{~Hz}$ & $0.7 \%$ \\
& $1 \mathrm{~Hz}-10 \mathrm{~Hz}$ & $1.5 \%$ \\
& $>10 \mathrm{~Hz}-20 \mathrm{~Hz}$ & $3 \%$ (only z-axis) \\
& $>20 \mathrm{~Hz}-40 \mathrm{~Hz}$ & $1.5 \%$ (x-, y-axis) \\
& $>40 \mathrm{~Hz}-160 \mathrm{~Hz}$ & $1.5 \%$
\end{tabular}

X-Axis calibration (cf. figure 15): The measurement deviation between CEA and SPEKTRA is within $0.1 \%$ all over the range. Deviation with PTB is within $2 \%$ at $1 \mathrm{~Hz}$, almost $1 \%$ up to $125 \mathrm{~Hz}$, and $2.5 \%$ at $160 \mathrm{~Hz}$.

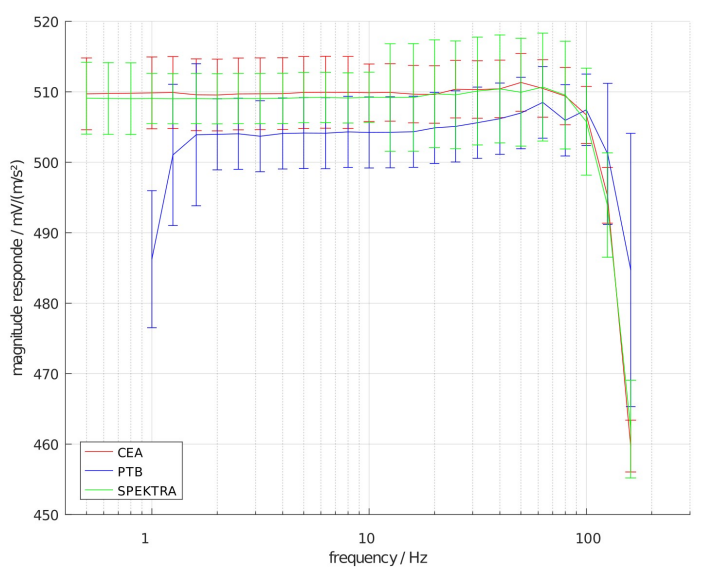

Figure 15. Magnitude response of the X-axis of the GeoSIG AC-73 (unit: $\mathrm{V} /\left(\mathrm{m}^{-2}\right)$ ).

Y-axis calibration (depicted in figure 16): The measurement deviation between CEA and SPEKTRA is within $0.2 \%$ up to $100 \mathrm{~Hz}$ and increases up to $3 \%$ at $160 \mathrm{~Hz}$. The deviation with PTB is similar with the x-axis up to $80 \mathrm{~Hz}$. At $160 \mathrm{~Hz}$, the deviation between the three calibrations is $8 \%$.

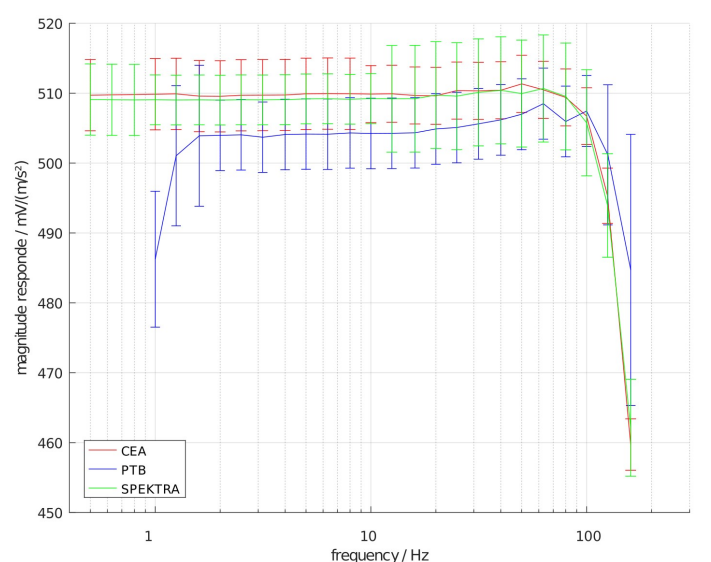

Figure 16. Magnitude response of the $y$-axis of the GeoSIG AC-73 (unit: $\mathrm{V} /\left(\mathrm{m}^{-2}\right)$ ).

Z-axis calibration (cf. figure 17): The measurement deviation between CEA and SPEKTRA is within $0.2 \%$ all over the range except of $30 \mathrm{~Hz}$. Deviation with PTB is within $2 \%$ at $1 \mathrm{~Hz}$, almost $1 \%$ up to $125 \mathrm{~Hz}$, and $1.5 \%$ at $160 \mathrm{~Hz}$.

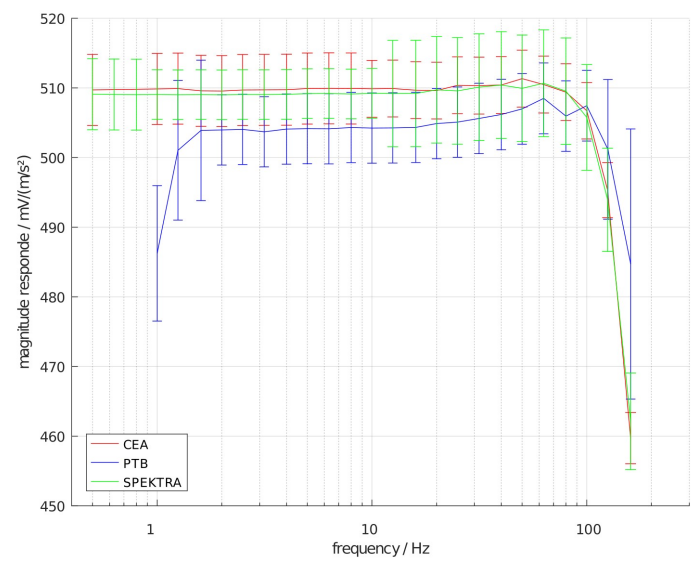

Figure 17. Magnitude response of the z-axis of the GeoSIG AC-73 (unit: $\left.\mathrm{V} /\left(\mathrm{m} \cdot \mathrm{s}^{-2}\right)\right)$.

\section{CONCLUSIONS AND OUTLOOK}

The mechanical calibration of large and heavy broadband sensors like seismometers is needed and is a challenge in the metrology domain of vibration. In order to improve knowledge in that field, and to evaluate the existing measuring capabilities, CEA, PTB and SPEKTRA calibrated one seismometer and one large accelerometer from $0.5 \mathrm{~Hz}$ up to $100 \mathrm{~Hz}$ and $160 \mathrm{~Hz}$, respectively. PTB used its multi-component acceleration exciter, CEA and SPEKTRA used CS18P calibration devices.

These first results show that the different types of exciters are capable to calibrate such sensors. They highlight that such measurements are very important to improve the knowledge in the real response of sensor as close as it behaves on field with ground motion as an input source. The calibration methodology and technical parameters effects like mounting, alignment need to be further analysed as the measurement uncertainty estimations. We also note that more types of exciters should be necessary to improve confidence in measurements. 


\section{REFERENCES}

[1] ISO 16063-11:1999, Methods for the Calibration of Vibration and Shock Transducers - Part 11: Primary vibration calibration by laser interferometry, International Organization for Standardization (ISO), Geneva, Switzerland, 1999.

[2] Prospect for low frequency seismometry. A report of the IRIS broadband seismometer workshop. Granlibakken, United States of America, 24-26 March 2004.

[3] Bureau International des Poids et Mesures (BIPM), Strategy 2017 to 2027 of the Consultative Committee for Acoustics, Ultrasound, and Vibration (CCAUV), 2017.

https://www.bipm.org/utils/en/pdf/CCAUV-strategydocument.pdf

[4] Larsonnier F. et al., Seismometer calibrations: comparison between a relative electrical method and a vibration exciter based absolute method, Proc. of IMEKO TC3, TC5, TC22 International Conferences, 2014, Cape Town, South Africa. https://www.imeko.org/publications/tc222014/IMEKO-TC22-2014-013.pdf

[5] Iwanczik M. et al., Primary calibration of geophysical velocity sensors and geophones. Proc of XXI IMEKO World Congress, 2015, Prague, Czech Republic. https://imeko.org/publications/wc-2015/IMEKOWC-2015-TC22-434.pdf

[6] Klaus L. et al., Seismometer calibration using a multicomponent acceleration Exciter. Journal of Physics: Conference Series, 2018.

DOI: $10.1088 / 1742-6596 / 1065 / 7 / 072043$ 